\title{
How to apply classical mechanics to the results of the video head impulse test?
}

\begin{abstract}
Functional evaluation of the vestibulo-ocular reflex (VOR) is one of the most important issues in the examination of the dizzy patient. The video Head Impulse Test (vHIT) has become an essential tool at the bedside and in the laboratory examination of the VOR. Most of vHITs devices only outputs the results of the head and eye movements based on velocity data and graphs, we consider that the position and acceleration data and graphs could have some clinical interest. With this interest we developed a standardized computer-based method to calculate position and acceleration graphs with the velocity data obtained from the vHIT. With the help of a specific computational development program a Graphical User Interface (GUI) was designed. Position and acceleration graphs were done using the classical mechanics methods. The GUI was tested measuring the head and eye displacements in normal and altered vHIT responses. The source files of the developed GUI are available on web: http://mlibra.com. The developed tool can be a useful tool to investigate some vHIT responses.
\end{abstract}

Keywords: vHIT; Position; Velocity; Acceleration; Classical mechanics; Integral calculus; Differential calculus; Saccades; Algorithm; Software
Volume I Issue I - 2014

\author{
Jorge Rey-Martinez,' Nicolas Perez- \\ Fernandez, ${ }^{2}$ Angel Ispizua-utierrez, ${ }^{3}$ Alberto \\ Urrutikoetxea-Sarriegui, ${ }^{4}$ Eusebi Matino, \\ Angel Batuecas-Caletrio 6 \\ 'Otolaryngology Unit Orlgipuzkoa, Clinica Quiron, Spain \\ 2Department of Otolaryngology, Clinica Universidad De \\ Navarra, Spain \\ ${ }^{3}$ Department of Otolaryngology, Hospital Universitario \\ Donostia, Spain \\ ${ }^{4}$ Department of Otolaryngology, Hospital Comarcal Del \\ Bidasoa, Spain \\ ${ }^{5}$ Department of Otolaryngology, Hospital General De \\ Catalunya, Spain \\ ${ }^{6}$ Department of ENT, University of Salamanca, Spain
} Orlgipuzkoa, Orlgipuzkoa, Clinica Quiron San Sebastian, Calle de Alkolea 7,200I2, San Sebastian, Spain, Email bendermh@hotmail.com

Received: November 23, 20I4 | Published: December 31, 2014 
ples per second, according with ICS vHIT factory specifications. ${ }^{4}$ For position vs. time the plot of the selected vHIT impulses the cumtrapz MATLAB function was used to estimate the position of the head and eye. The cumptrapz function estimates the integral of a graphic using the trapezoidal approximation method (Figure 2), which is an accepted method to calculate the area under the curve in periodic functions. ${ }^{5}$

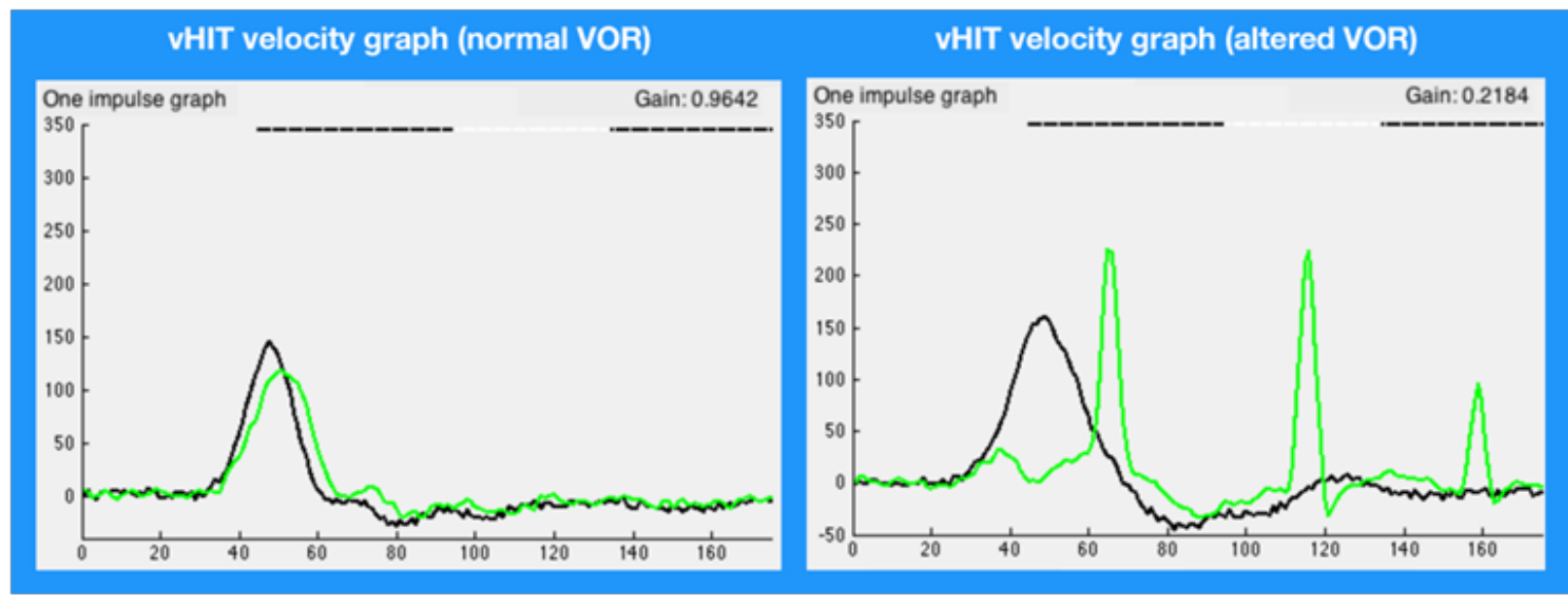

Figure I vHIT velocity graphs. On the left graph the head velocity (plotted in black) obtained with the head impulse is followed by a similar (in absolute values) eye movement (plotted in green). In the right plot, eye velocity does not follow the head movement and three movements are recorded (refixation saccades) after the head movement has been finished.Y-axis: velocity in deg./s. X-axis: time in (hardware) samples.

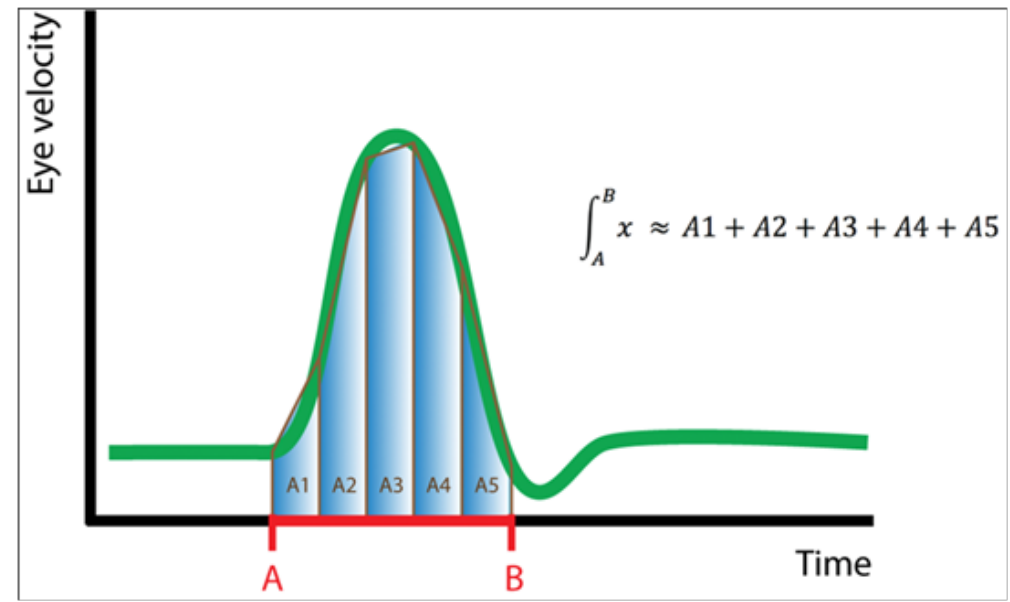

Figure 2 The trapezoidal approximation method. For the A-B time interval, the area under the eye velocity curve (plotted in green) can be approximated with the sum of the areas of a finite number of trapezoids (AI,A2,A3, A4 and A5) contained in the A-B interval.

The acceleration vs. time plot was obtained from the variation rate of velocity using the diff MATLAB function. With the calculated data a Graphical User Interface (GUI) was designed, and named Integral Calculus Tool. As shown in Figure 2 one part of this GUI contains the six movement plots, with the tree graphs (velocity, acceleration and position) for head and eye movement. An interval selection tool was also implemented in this GUI, to allow the partial analysis of a determined time interval of the original velocity graphs. To test the Integral Calculus Tool we used 10 anonymized vHIT responses collected from the database of vHITs from the hospital of first author of this study; an experimented explorer performed these vHIT. In normal subjects and in patients, 5 horizontal impulses were selected and in the case of the patients these had to include saccades; patients with spontaneous Nystagmus (to either side) were excluded. All the selected impulses had similar head impulse displacement (7.5-9.5 degrees).

For each impulse the area under curve for the head impulse time interval was compared with the area under curve for the normal eye response (Figure 3). A similar approach was done for the saccades detected (Figure 4\&5). The data collected for this study was obtained under informed consent, signed by each participant, authorizing the use of his results in this study. Data for this study were stored in a file as anonymous content (Identifiable patient information was not included in the database used in this study). These terms are in accordance with Spanish regulation applicable to the protection of personal data (LOPD 15/1999).

The design of this retrospective study was carried out following the guidelines of the Declaration of Helsinki of the World Medical Association on Ethical Principles for Medical Research Involving Human Subjects. The ethics committee of each participant hospital accepted the protocol of this study. In compliance with the regulation currently in force for research in the Spanish state (ORDEN SAS / 3470/2009) for the type of study (retrospective, observational and anonymized) performed in this paper the design of a study protocol in accordance with Declaration of Helsinki is an ethically valid methodology. According this regulation, for studies of this type, the approval or monitoring by the regional clinical research ethics committee is not required. 


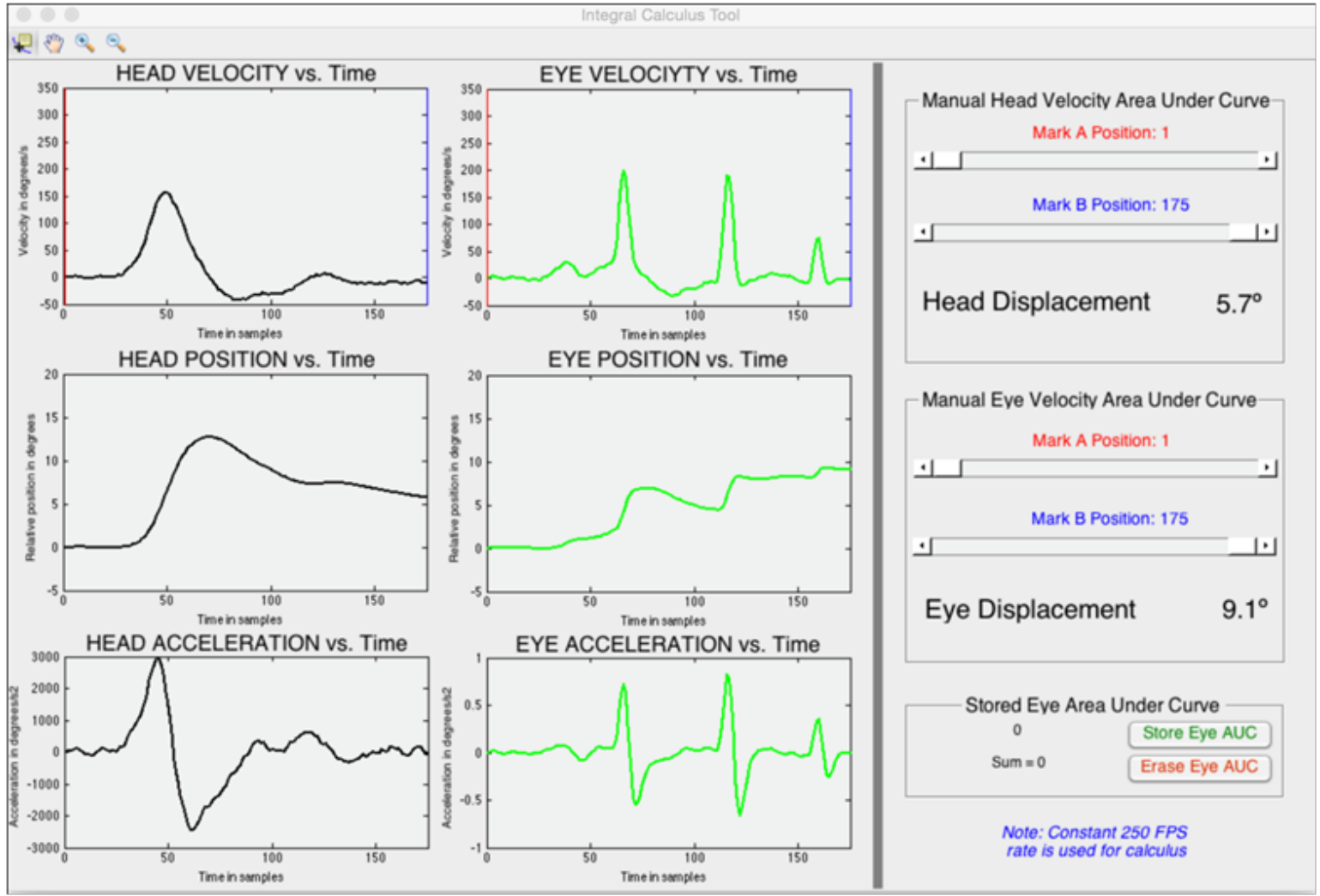

Figure $3 \mathrm{Head}$ and eye area under curve measurement in normal response impulses. The first row of images is the calculation of the area under curve during the head impulse. The second row of images is the calculation of the area under curve during the eye response. Calculated displacements of the head and the eye are the same, 7.8 degrees.

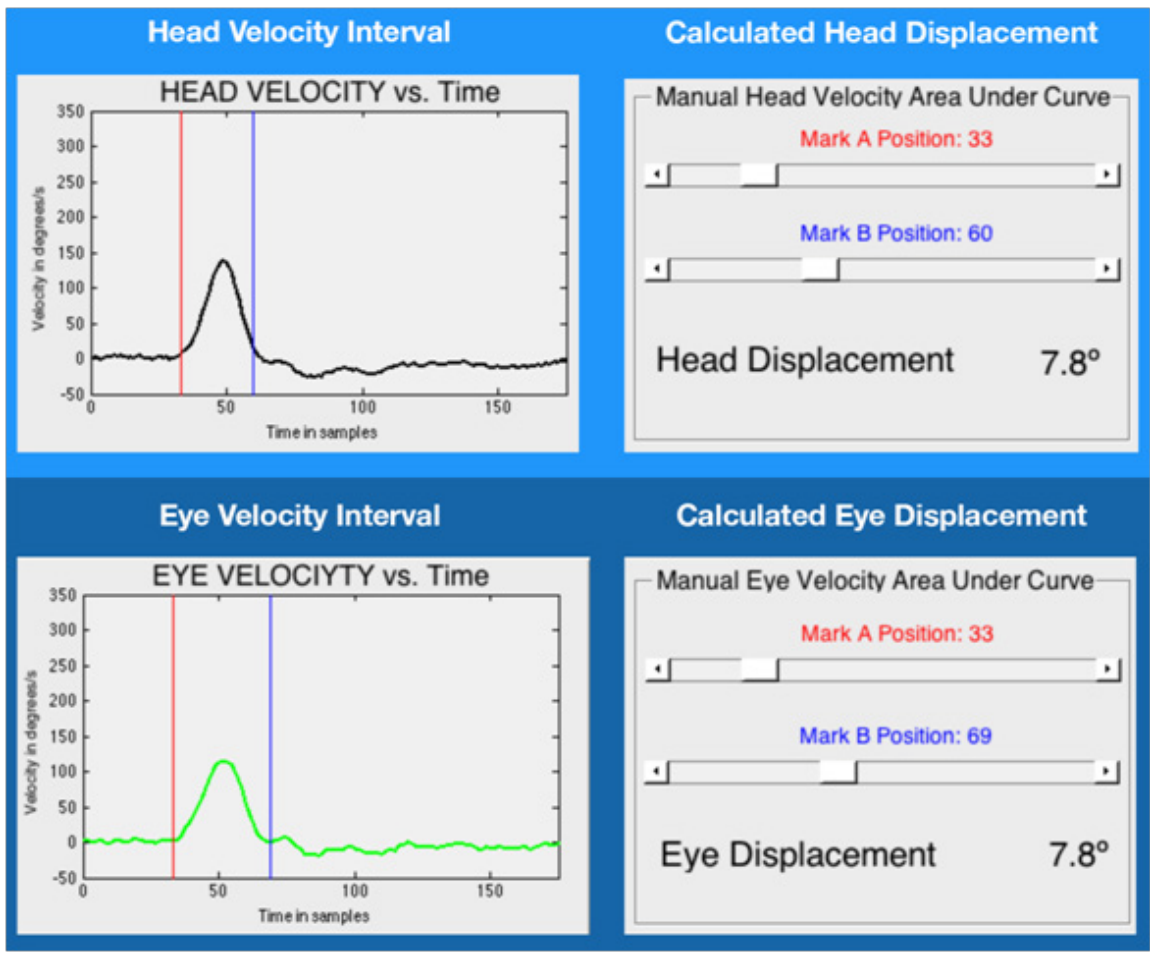

Figure $4 \mathrm{Head}$ and eye area under curve measurement in altered response impulses. The first row of images is the calculation of the area under curve during the head impulse. The second row of images is the calculation of the area under curve during the first eye response. The third row of images is the calculation of the area under curve during the second eye response. The fourth row of images is the calculation of the area under curve during the third eye response. The accumulative calculated eye displacement is similar to head displacement, $11.9 \approx 12.5$ degrees. 


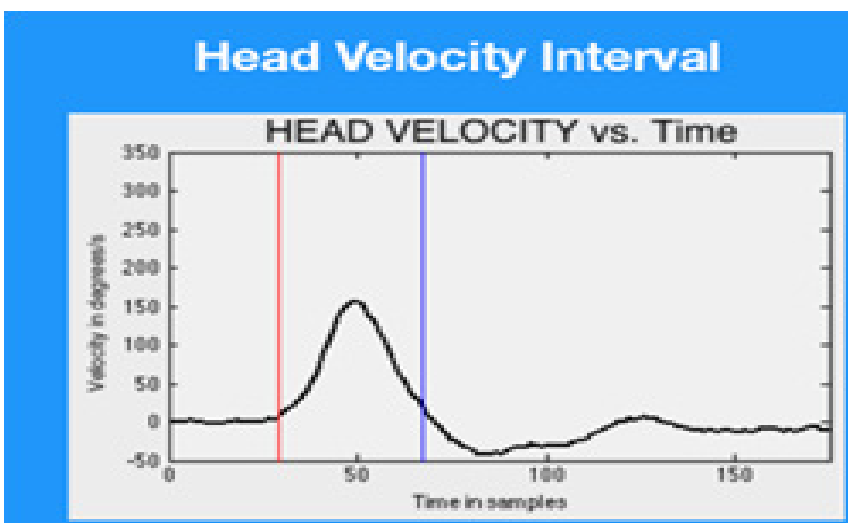

1st. Eye Velocity Interval

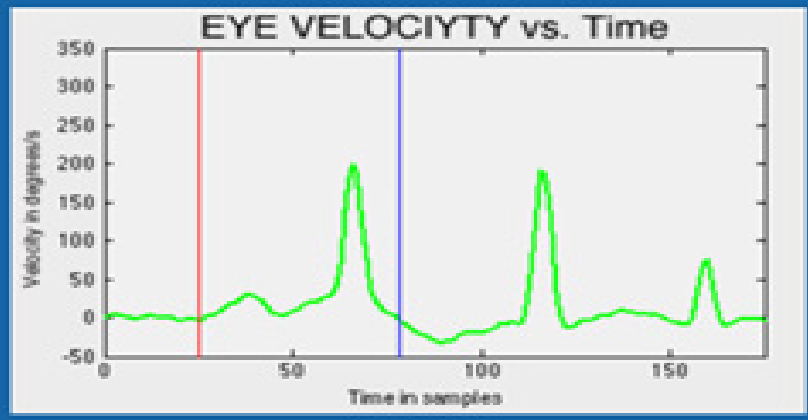

2nd. Eye Velocity Interval

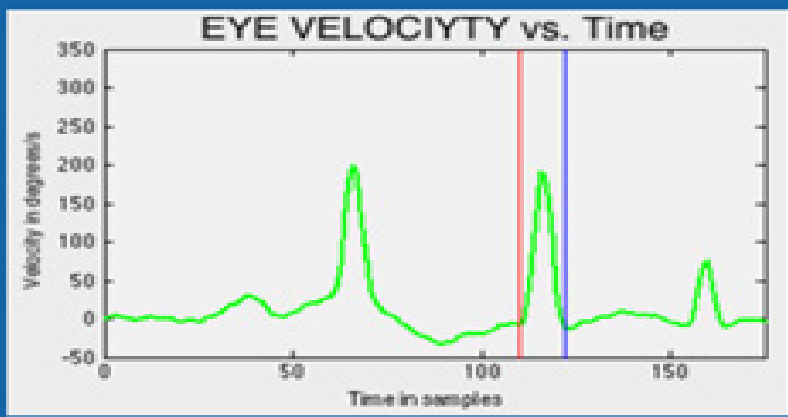

3rd. Eye Velocity Interval

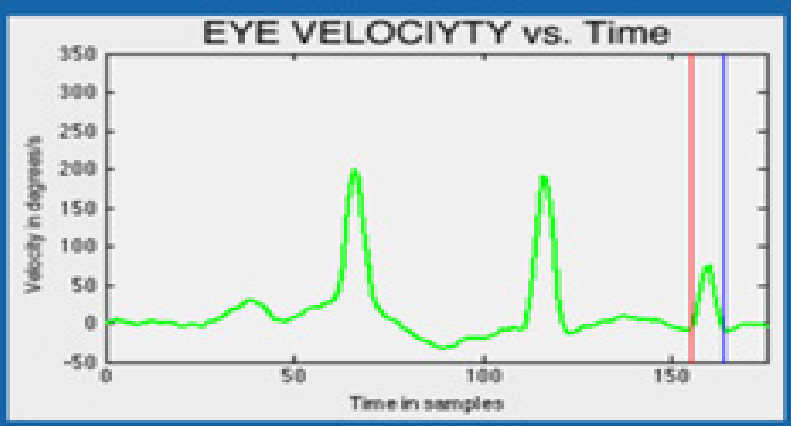

Calculated Head Displacement

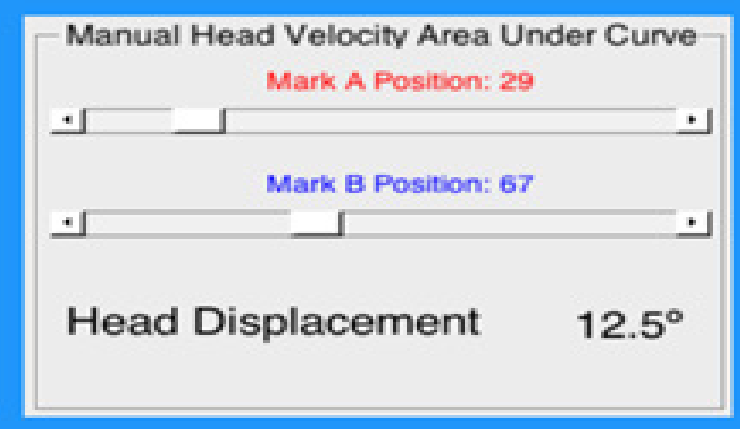

\section{1st. Calculated Eye Displacement}

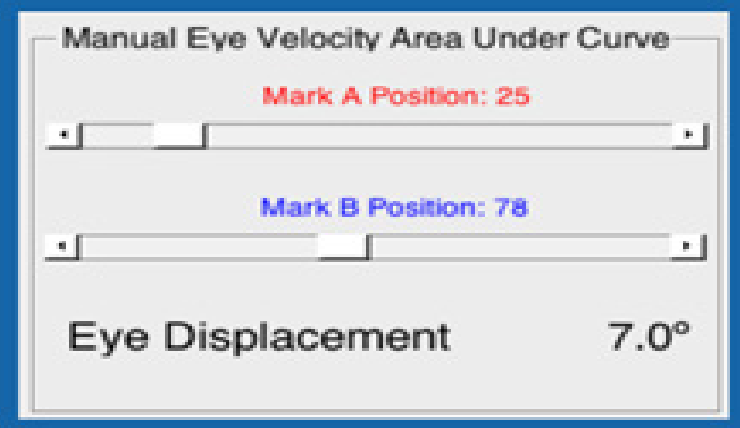

2nd. Calculated Eye Displacement

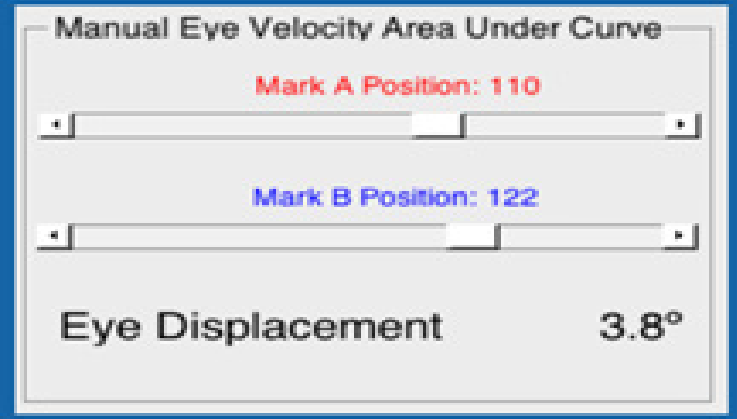

3rd. Calculated Eye Displacement

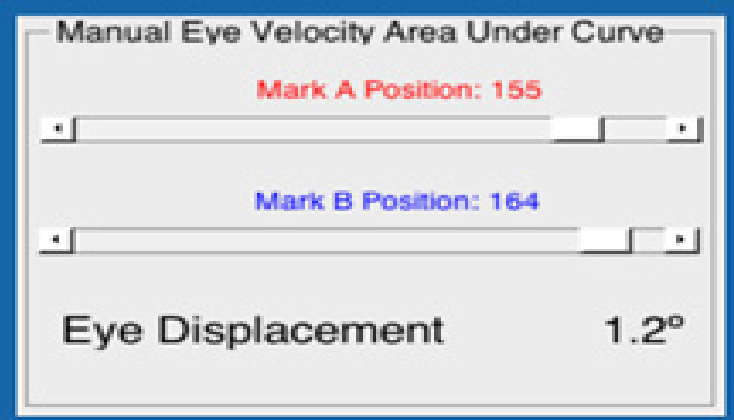

\section{Accumulated Eye Displacement $=11.9^{\circ}$}

Figure 5 Accumulated Eye Displacement. 


\section{Results and discussion}

As result of this study an Integral Calculus Tool GUI was developed using MATLAB. The GUI was fully operative and its main functions are: velocity vs. time plots, position vs. time plots and acceleration vs. time plots for eye and head movement, interval selection for area under curve calculation, accumulative area under curve calculation. In the test of the Integral Calculus Tool with normal subjects $(n=5)$ the same displacement was measured in head and eye movements. Head displacement (area under impulse curve $)=8.36$ degrees (SD: 0.23), eye displacement (area under impulse curve) $=8.17$ degrees (SD: $0.52)$. In the test with patients $(n=5)$ with detected saccades, the same displacement was measured in head and eye movements. Head displacement (area under impulse curve) $=7.98$ degrees (SD: 0.35), eye displacement (area under impulse and area under post-impulse) $=7.52$ degrees (SD: 1.03). These position and acceleration data obtained with the Integral Calculus Tool GUI were in the same value ranges of the values published by other authors. ${ }^{6}$

The advantages of knowing the acceleration or the position data of the eye and head measured in the VOR responses have been reported in experimental researches. ${ }^{7}$ The acceleration data are frequently used in other similar exploration techniques, ${ }^{8}$ but some of these techniques are difficult to use in habitual clinical practice. With the GUI developed in this article, all this data could be obtained and analyzed in the clinical explorations where ICS - video head impulse test is used, without any other additional equipment. The future implications or advantages of the clinical application of this enhanced methodology are actually unknown.

One of the main limitations of our tool is that during the testing a constant sample rate of 250 was assumed; however the data stored in the XML file is not always constant as the vHIT software automatically rejects low sample rates to minimize registration artifacts. As such this "near to" 250 sample rate could affect to the position and acceleration calculations. The ICS vHIT fabricant recommends using the RAW data file for the position calculation, in this RAW file the real time is registered for each measurement. The problem with this RAW format is that all the data recorded during the test is included. The RAW file is a large record file, inside which all files are recorded: the eye an head movements occurred during the well-performed impulses, the not well-performed impulses and even any other movement occurred during the entire test time. To design an automated analysis method based on RAW files, a new impulse recognition algorithm is needed. This algorithm must analyze the entire RAW data selecting the well-performed impulses. The implementation of this impulse recognition algorithm exceeds the proposed methodology for this study. To solve this, we decided to use the XML file format; in which all the data stored is pre-processed by the ICS vHIT software algorithm to select the well-performed impulses. ${ }^{9}$

The possibility of determining intervals to analyze the graphics is an important feature of the developed GUI. With this feature the user can select and specifically compare the movement during head impulse and eye movements that want to analyze. In certain records may exist head movements (such as overshoot) that does not interest us to analyze, at other times the vHIT can record very low speed movements appeared after the head impulse that the user neither want to analyze. The interval selection feature makes possible that all these movements can be analyzed separately. It has been shown that saccades are one type of compensatory responses aimed to reduce retinal smear during head movements in patients with a deficient VOR. ${ }^{10,11}$ With the methodology developed in this article we can measure the efficiency of this compensatory mechanism from the data obtained using the vHIT in a small sample of impulses. This methodology can be used in further studies to characterize the saccadic responses on patient with altered VOR. This Integral Calculus Tool GUI has been incorporated in our vHIT advanced analysis software: HIT Cal. The HIT Cal source files are available on web: http://mlibra.com.

\section{Conclusion}

In this article we describe a standardized software tool for vHIT position and acceleration vs. time graphs calculation. The developed tool can be a useful tool to investigate some VOR responses.

\section{Acknowledgments}

None.

\section{Conflicts of interest}

The authors declare that there is no conflicts of interest.

\section{References}

1. Curthoys IS. The interpretation of clinical tests of peripheral vestibular function. Laryngoscope. 2012;122(6):1342-1352.

2. Weber KP, Aw ST, Todd MJ, et al. Head impulse test in unilateral vestibular loss: vestibulo-ocular reflex and catch-up saccades. Neurology. 2008;70(6):454-463.

3. Isaac Newton. Philosophiae naturalis principia mathematica, 1687.

4. No authors listed. ICS Impulse ${ }^{\circledR}$ system Performance Characteristics, 2014.

5. Weideman JAC. Numerical Integration of Periodic Functions: A Few Examples. The American Mathematical Monthly. 2002;109(1):21-36.

6. Curthoys IS, MacDougall HG, Manzari L. Clinical application of a new objective test of semicircular canal dynamic function - the video head impulse test (vHIT), A safe, simple and fast clinical vestibular test, 2012.

7. Cullen KE, Minor LB, Beraneck M, et al. Neural substrates underlying vestibular compensation: Contribution of peripheral versus central processing. J Vestib Res. 2009;19(5-6):171-182.

8. Weber KP, Aw ST, Todd MJ, et al. Inter-ocular differences of the horizontal vestibulo-ocular reflex during impulsive testing. Prog Brain Res. 2008;171:195-198.

9. Wendy Crumley-Welsh. ICS Impulse - Revolutionizing Vestibular Assessment. Audiology Online, 2013.

10. Batuecas-Caletrio A, Santacruz-Ruiz S, Munoz-Herrera A, et al. The vestibulo-ocular reflex and subjective balance after vestibular schwannoma surgery. Laryngoscope. 2014;6:1431-1435.

11. Mc Dougall HG, Curthoys IS. Plasticity during vestibular compensation: The Role of Saccades. Front Neurol. 20123:1-9. 